\title{
Systems Biology and Physiology Reports in 2021: a yearly report
}

\section{by Mikhail A. Panteleev ${ }^{1-3} \#$}

1. Faculty of Physics, Lomonosov Moscow State University, 1/2 Leninskie gory, Moscow, 119991, Russia

2. Center for Theoretical Problems of Physico-Chemical Pharmacology, Russian Academy of Sciences, 30 Srednyaya Kalitnikovskaya str., Moscow, 109029, Russia

3. National Medical Research Center of Pediatric Hematology, Oncology and Immunology named after Dmitry Rogachev, 1 Samory Mashela St, Moscow, 117198, Russia

\section{\# Correspondence: editor@sbpreports.com}

Received: 30.12.2021

Accepted: $\mathbf{3 0 . 1 2 . 2 0 2 1}$

Published: 31.12.2021

DOI: 10.52455/sbpr.01.202104014

\section{Dear All,}

I am happy to greet all readers, editors, reviewers, staff members and publishers of SBPReports on the eve of year 2022. I thank all of you for your efforts in creating this journal and making it work despite all the challenges faced by a new independent journal in the age dominated by huge publishers, research societies and citation systems. Please accept my best wishes for the year 2022 in your professional and personal undertakings!

Where do we stand now, and what are our goals for 2022?

The scope and the style of the journal have become somewhat crystallized over this year. After a year of development, SBPReports boasts a highly professional and dedicated editorial board formed by experts in the systems biology field from Belarus, Canada, China, India, Russia, UK and USA. The three major sections of the edition are related to the three major levels of understanding of the biological systems: molecular $[1,2]$, cellular $[3,4]$, and organism $[5,6]$ levels. The papers published in the four issues during this year include original articles $[1,2,4,6]$, reviews [5,7-9], and methodological studies $[10,11]$. In addition, two large abstract collections Systems Biology and Systems Physiology Congress 2021 and International Congress devoted to the Memory of the Founders of Biophysics Department of Physics Faculty of Lomonosov Moscow State University professor L.A. Blumenfeld and professor S.E. Shnoll have been published during this year as proceedings of two important international conferences in our field; the journal also served as a platform for the conference planning.

\section{The following goals could be indicated for 2022.}

1. We need to work on increasing the diversity of the subjects (which are presently dominated by immunology and hematology) and of the geography of the submissions.

2. The first essential steps towards inclusion in the citation systems (first of all, Russian Science Citation
Index and Directory of Open Access Journals) need to be undertaken.

3. finally, and most importantly, SBPReports will continue to publish novel and significant research to contribute to the knowledge of the complex biological systems.

Thank you for being with us this year, there is so much more to come.

On behalf of the SBPReports editorial board, Mikhail Panteleev

\section{References}

1. Korobkin J, Garcia A, Sveshnikova A. A minimal mathematical model of neutrophil pseudopodium formation during chemotaxis. Systems Biology and Physiology Reports 2021;1:6. https://doi.org/10.52455/ sbpr.01.202103012.

2. Garzon Dasgupta A. STIM1-ORAI1 direct interaction cannot govern store-operated calcium entry (SOCE) in platelets. Systems Biology and Physiology Reports 2021;1:11. https://doi.org/10.52455/sbpr.01.202102012.

3. Galkina S, Balabin F. On calcium fluorophore's impact in platelet signaling studies. Systems Biology and Physiology Reports 2021;1:10. https://doi.org/10.52455/ sbpr.01.202104012.

4. Tabachnik A. Presence of PI-rich vesicles is required for the PLC $\zeta$ activation according to mathematical modeling. Systems Biology and Physiology Reports 2021;1:17. https://doi.org/10.52455/sbpr.01.202102013.

5. An O, Martyanov A, Panteleev M. Immune thrombocytopenia: what can the systems biology and systems physiology offer? Systems Biology and Physiology Reports 2021;1:1. https://doi.org/10.52455/ sbpr.01.202104011.

6. Stepanyan M, Martyanov A, An O, Boldova A, Roumiantsev $S$, Rumyantsev $A$, et al. A strong correlation exists between platelet consumption and platelet hyperactivation in COVID-19 patients. Pilot study of the patient cohort from $\mathrm{CCH}$ RAS Hospital (Troitsk). Systems Biology and Physiology Reports 2021;1:1. https://doi.org/10.52455/sbpr.01.202102011.

7. Sveshnikova A, Stepanyan M, Panteleev M. Platelet functional responses and signalling: the molecular relationship. Part 1: responses. Systems Biology and Physiology Reports 2021;1:20. https://doi.org/10.52455/ sbpr.01.202101014.

8. Asadov A, Chudinov I, Nechipurenko D. In vitro models 
of thrombosis and hemostasis. Systems Biology and Physiology Reports 2021;1:23. https://doi.org/10.52455/ sbpr.01.202102014.

9. Grigorova I. Overview of the neutralizing antibody and memory B cell response kinetics in SARS-CoV-2 convalescent and/or mRNA vaccinated individuals. Systems Biology and Physiology Reports 2021;1:1. https://doi.org/10.52455/sbpr.01.202103011.

10. Kokhan A, Zdanevich S, Prokofev I, Gorudko I, Shamova E, et al. Patch-clamp technique for studying ion channels in activated platelets. Systems Biology and Physiology Reports 2021;1:3. https://doi.org/10.52455/ sbpr.01.202101012.

11. Alexandrova $\mathrm{V}$, Anisimov M, Eltsov I, KilinaA, Lopanskaia I, Makarova L, et al. Avoiding common problems with statistical analysis of biological experiments using a simple nested data simulator. Systems Biology and Physiology Reports 2021;1:12. https://doi.org/10.52455/ sbpr.01.202101013. 